\title{
The Effects of Lifestyle and/or Vitamin D Supplementation Interventions on Pregnancy Outcomes: What Have We Learned from the DALI Studies?
}

\author{
Jürgen Harreiter ${ }^{1}$ • Gernot Desoye ${ }^{2}$ - Mireille N. M. van Poppel ${ }^{3}$. Alexandra Kautzky-Willer ${ }^{1}$ • Fidelma Dunne ${ }^{4}$. \\ Rosa Corcoy ${ }^{5,6,7}$ - Roland Devlieger ${ }^{8}$. David Simmons ${ }^{9,10}$ - on behalf of the DALI Consortium - Juan M. Adelantado • \\ Peter Damm • Elizabeth Reinhardt Mathiesen • Dorte Moeller Jensen • Lise Lotte T. Anderson • Annunziata Lapolla • \\ Maria G. Dalfrà • Alessandra Bertolotto • Ewa Wender-Ozegowska • Agnieszka Zawiejska • David J. Hill • Frank J. Snoek
}

(C) Springer Science+Business Media, LLC, part of Springer Nature 2019

\begin{abstract}
Purpose of Review The DALI (vitamin D and lifestyle intervention in the prevention of gestational diabetes mellitus (GDM)) study aimed to prevent GDM with lifestyle interventions or Vitamin D supplementation (1600 IU/day). This review summarizes the learnings from the DALI studies among pregnant women with a BMI $\geq 29 \mathrm{~kg} / \mathrm{m}^{2}$.

Recent Findings Women diagnosed with GDM earlier in pregnancy had a worse metabolic profile than those diagnosed later. A combined physical activity (PA) and healthy eating (HE) lifestyle intervention improved both behaviours, limited gestational weight gain (GWG) and was cost-effective. Although GDM risk was unchanged, neonatal adiposity was reduced due to less sedentary time. Neither PA nor HE alone limited GWG or GDM risk. Fasting glucose was higher with HE only intervention, and lower with Vitamin D supplementation.

Summary Our combined intervention did not prevent GDM, but was cost-effective, limited GWG and reduced neonatal adiposity.
\end{abstract}

Keywords Gestational diabetes mellitus $\cdot$ Obesity $\cdot$ Prevention pregnancy $\cdot$ Lifestyle intervention $\cdot$ Vitamin D

\section{Introduction}

Gestational diabetes mellitus (GDM) is a common pregnancy complication and was reported in 2012 to have a European prevalence of $2-6 \%[1 \bullet]$. Obesity is a well-known risk factor for GDM [2] and the prevalence of both GDM and obesity in pregnancy is increasing [2-4].Women with GDM have 7.5fold higher risk for the development of type 2 diabetes mellitus (T2DM), and a nearly 2-fold increased risk of cardiovascular disease (CVD) $[5 \cdot, 6]$. A nearly 3 -fold increased CVD risk was found in those women with a history of GDM who developed T2DM [7]. Both GDM and obesity are associated with adverse pre- and perinatal outcomes,

This article is part of the Topical Collection on Diabetes and Pregnancy

David Simmons

da.simmons@westernsydney.edu.au

Extended author information available on the last page of the article including hypertension, pre-eclampsia, macrosomia, shoulder dystocia, operative delivery, stillbirth, jaundice and neonatal hypoglycaemia, as well as higher risk of metabolic disorders in the offspring $[2,8]$.

Outside pregnancy, lifestyle interventions have been associated with significant reductions in T2DM development, including among women with past GDM [9••]. Furthermore, several studies have reported that such lifestyle intervention strategies in the prevention of T2DM in high-risk populations are cost-effective $[10,11]$. Due to pathophysiological similarities between GDM and T2DM, it is reasonable to postulate that lifestyle interventions might also be effective during pregnancy to prevent GDM, and that these might be cost-effective.

Similarly, systematic reviews have found an association between risks of hyperglycaemia, including T2DM, and vitamin $\mathrm{D}$ deficiency in non-pregnant adults, with lower risk of T2DM and lower glucose levels found in subjects with high 25-hydroxyvitamin D levels [12, 13]. However, results from vitamin D supplementation in patients with T2DM are heterogeneous due to poor quality 
and low participant numbers. Only modest reductions in $\mathrm{HbA} 1 \mathrm{c}$ and no significant differences in fasting glucose levels have been shown compared with placebo [14]. Recently, the D2d trial including over 2400 patients found that patients with prediabetes, defined as meeting two of three criteria for prediabetes (i.e. fasting glucose 100$125 \mathrm{mg} / \mathrm{dl}, 2-\mathrm{h}$ postprandial glucose $140-199 \mathrm{mg} / \mathrm{dl}$ or HbA1c 5.7-6.4\%) who received vitamin D3 supplementation at a dose of $4000 \mathrm{IU} /$ day had comparable risk for progression to T2DM (HR 0.88; 95\% CI 0.75-1.04; $p=.12$ ) as the placebo group after a 2.5-year follow-up [15]. Since in pregnancy, associations had been found between low vitamin D levels and higher risk of GDM (OR 1.85, 1.47-2.32), higher fasting plasma glucose and higher HOMA-IR [16], vitamin D supplementation was thought to be another intervention method to prevent the progression of GDM in pregnancy at the time the DALI study was designed.

The DALI (vitamin D and lifestyle intervention in the prevention of GDM) studies were established to describe the epidemiology of GDM in Europe and to identify intervention strategies to reduce GDM risk. At the time, the strategies chosen to test to reduce GDM risk were healthy eating (HE) and physical activity (PA) alone, or together, and vitamin D supplementation at a high enough dose to ensure most obese women would become replete. The DALI project has generated 15 publications dealing with the epidemiology of GDM across Europe, effects of lifestyle intervention or vitamin D supplementation on GDM risk and the offspring, factors mediating intervention effects, cost-effectiveness of GDM prevention methods, mental health in pregnancy and potential unintended side effects of lifestyle intervention. This paper summarises the learnings to date, in the context of other publications since DALI commenced in 2012.

\section{DALI Study Design}

The DALI studies were conducted between 2012 to 2015 in 11 centres across nine countries in Europe (Austria, Belgium, Denmark, Ireland, Italy, Poland, Spain, Switzerland, the Netherlands, United Kingdom). The interventions were tested in 3 multicentre RCTs, one a pilot and two with a factorial design [17]. The trial was registered under the number ISRCTN7059583, was performed according to the declaration of Helsinki and all local ethics committees approved the study. Participating women were included before 20 weeks of gestation, with age above 18 years, a pre-pregnancy BMI of $\geq$ $29 \mathrm{~kg} / \mathrm{m}^{2}$ and a singleton pregnancy. Data from all DALI sites were initially analysed to define the BMI cutoff of $29 \mathrm{~kg} / \mathrm{m}^{2}$ in order to allow recruitment of sufficient women as lesser rates of maternal obesity were found in some participating sites [18]. A diagnosis of GDM at the baseline oral glucose tolerance test (OGTT), pre-existing diabetes, chronic medical conditions, a psychiatric disorder, walking inability of $\geq$ $100 \mathrm{~m}$, complex diet requirement and language barriers in the major language of the country of recruitment were exclusion criteria. The International Association of Diabetes and Pregnancy Study Group (IADPSG)/World Health Organization (WHO) 2013 criteria were used to diagnose GDM, which was assessed by OGTT before 20, at 24-28 and 35-37 weeks of gestation (venous plasma glucose: fasting $\geq 5.1 \mathrm{mmol} / \mathrm{L}, 1 \mathrm{~h} \geq 10 \mathrm{mmol} / \mathrm{L}, 2 \mathrm{~h} \geq 8.5 \mathrm{mmol} / \mathrm{L}$ ).

\section{DALI Lifestyle Intervention}

Participating women were either randomised to healthy eating, physical activity, healthy eating and physical activity (HE\&PA) or usual care (UC) in the main trial, whereas in the pilot study, only the three lifestyle interventions were tested $[19 \bullet \bullet, 20]$. The pilot study was performed to test study processes and to detect limitations and weaknesses, which needed to be tackled before starting the main trial [21•]. In the DALI lifestyle trial, seven healthy eating and/or 5 physical activity messages (Table 1) were conveyed through lifestyle coaches after randomisation. All interventions were aiming to achieve a total gestational weight gain of $5 \mathrm{~kg}$ in pregnancy, which is the lower limit of the recommended gestational weight gain in obese pregnant women according to the Institute of Medicine [22•].

The lifestyle intervention was based on behavioural and empowerment techniques using motivational interviewing methods [23], delivered in 5 face to face meetings with 4 telephone calls/electronic messaging in between. The HE

Table 1 DALI lifestyle intervention messages

Healthy eating messages

1. "Replace sugary drinks"

2. "Eat more non-starchy vegetables"

3. "Increase fibre consumption"

4. "Watch portion size"

5. "Eat more protein"

6. "Reduce fat intake"

7. "Eat less carbohydrates"

Physical activity messages

1. "Be active every day"

2. "Sit less"

3. "Build your strength"

4. "Take more steps"

5. "Be more active at weekends"

(Reproduced from: Jelsma JG, et al. BMC Pregnancy Childbirth. 2013;13:142. doi: https://doi.org/10.1186/1471-2393-13-142; Creative Commons user licence https://creativecommons.org/licenses/by/4.0/) [17] 
messages delivered by the coaches were based on food quality and quantity with focus on intake of complex rather than simple carbohydrates, higher intake of protein and fibre, and reduced fat consumption on the one hand and a focus on portion size to reduce total daily calorie intake on the other hand. The PA intervention tried to increase physical activity and reduce sedentary behaviour by promotion of aerobic and resistance physical activity. Participating women received a participant handbook and educational materials, as well as a pedometer (Digiwalker SW-200; Yamax, Tokyo, Japan), and a flexible elastic Dyna-Band (Thera-Band, Akron, $\mathrm{OH}$ ). A mobile technology approach using a handheld electronic device (HTC HD7, HTC Corporation, Taiwan) was used to support our coaches [24].

\section{Vitamin D Intervention Trial}

In the vitamin D trial of DALI, participating women were randomised to 4 different groups [25••]. The groups were either vitamin $\mathrm{D}$ or placebo alone or vitamin $\mathrm{D}$ or placebo with lifestyle advice, which was a combination of HE\&PA. Similar cutoff levels of vitamin D in pregnancy to nonpregnant adults were used and thus we aimed to exceed a vitamin D3 level of $\geq 50 \mathrm{nmol} / 1$ at delivery [25••].Vitamin D3 was supplemented in a dose of 1600 IU daily starting in early pregnancy before 20 weeks of gestation [25••]. The dose of $1600 \mathrm{IU} /$ day was chosen to account for a total intake of $2000 \mathrm{IU} /$ day with supplements considering a high proportion of women taking multivitamins in pregnancy with an average vitamin D3 content of 400 IU per tablet. Initially, in the pilot study, a vitamin D dosing and safety trial was planned, which was not executed due to external research results published shortly before pilot start and demonstrating a safe and sufficient application of vitamin D in pregnancy in higher doses than planned in DALI [26•]. Furthermore, the upper tolerable intake in pregnancy was increased to $4000 \mathrm{IU} /$ day at the same time [27]. Vitamin D3 (Devaron®) with a dose of $400 \mathrm{IU}$ per tablet and identical placebo tablets were produced by Vemedia (Diemen, Netherlands). In the vitamin D trial, the beneficial effects of supplementation of vitamin D3 with or without lifestyle intervention on prevention of GDM assessed by fasting glucose, HOMA IR and weight gain compared with placebo with and without lifestyle intervention were tested as well as safe application.

\section{Process Evaluation and Fidelity}

In a process evaluation of the interventions in the DALI pilot study, relevant knowledge about the implementation process of lifestyle intervention delivered with behavioural techniques based on motivational interviewing in obese pregnant women was gathered [20]. DALI was a Pan-European trial and thus different languages and cultures made it challenging to deliver the same intervention messages to the participating women across all sites and so a process evaluation was important to find restrictions and limitations as well as positive aspects of the intervention. When using motivational interviewing techniques, a quality assessment of the intervention is recommended to identify the effect of the interventions on the outcome [28]. A majority of women (63\%) received all intervention sessions and reported high satisfaction with several intervention aspects (ratings 7.6-9.2 out of 10 points for maximum satisfaction). However, across sites high variability of motivational interviewing competency of lifestyle coaches was reported. Several global motivational interviewing scores were assessed to evaluate fidelity and beginning proficiency or almost beginning proficiency was reached by the coaches, mostly. However, the overall motivational interviewing scores were below expert cutoffs. Associations between the process elements fidelity and dose, which are a level of coaching proficiency or number of contacts, and gestational weight gain were not detected. A need for further studies in this field to find solutions to attract non-responders as well as increased feedback possibilities and recording facilities throughout the study was reported to be potentially helpful to increase motivational interviewing fidelity [20].

Preferences and perceived barriers of the DALI participants were analysed. Obese women indicated that offspring health was a main driver to participate in an intervention study, but barriers such as tiredness, pain or physical limitations and lack of time due to child care were named as one of the main barriers for physical activity [29]. Proper background knowledge about risk in pregnancy was rated important, as well as flexibility and individual modification of the intervention and partner support.

\section{Epidemiology of GDM}

In DALI, a total GDM prevalence of $39 \%$ was detected across early, mid and late gestation [30••]. Former prevalence data found tremendously lower GDM rates of 2-6\% in a retrospective analysis and including women of all BMI categories [ $[\bullet]$. These differences might be explained through different screening modalities (universal vs. risk-based) and different diagnostic criteria as well as poor clinical awareness [1•]. In DALI, with identical protocols for screening and diagnosis in all countries, higher rates were detected in Northern Europe with a prevalence of $52 \%$ in Denmark, whereas lower rates were found in Western Europe with a prevalence of 24\% and $25 \%$ in the UK and Ireland [30••]. These large variations between countries were unexpected. A high rate of GDM was found in early pregnancy with $24 \%$ (prevalent GDM, i.e. women presenting with GDM already at first examination), with a further $14 \%$ and then $13 \%$ new cases (incident GDM, i.e. developing GDM de novo during pregnancy) at 24-28 and 35-37 weeks respectively. As many European countries were 
involved, and different clinical routines were in place, there is a risk that the differences in GDM prevalence might be explained by different sample processing. To diminish the chance of such variation in sample handling and collection, strict standard operating procedures were defined before trial start, and tested in a pilot study to assure identical processing across all countries. All samples were stored immediately at $20{ }^{\circ} \mathrm{C}$ or below and centrally analysed in the ISO-certified laboratory of the Medical University of Graz. Nonmodifiable risk factors for the development of GDM (women developing GDM vs. those maintaining a normal OGTT respectively) [30••, 31] included recruitment site, lower maternal height, previous GDM (16.5\% vs. $7.9 \%, p<0.01)$, past babies with congenital malformations (6.4\% vs. $3.3 \%$, $p<0.05)$ and previous macrosomia (31.4\% vs. $17.9 \%$, $p<0.01)$. Potentially modifiable risk factors included larger neck circumference and higher resting heart rate [31]. Pregnancy outcomes did not differ overall between women with normal glucose tolerance and women with GDM developing mid-late pregnancy.

\section{Heterogeneity of GDM}

Compared with women with a later diagnosis of GDM, women diagnosed with GDM before 20 weeks of gestation had higher insulin resistance, BMI, waist circumference, blood pressure and heart rate as well as lipids, which are all features of the metabolic syndrome [32•]. Nearly $80 \%$ of these women were identified by increased fasting glucose. The presence of early GDM (compared with early normal glucose tolerance) was associated with higher pre-pregnancy BMI (OR 1.05, $95 \%$ CI $1.00-1.10$, per $\left.\mathrm{kg} / \mathrm{m}^{2}\right)$ and sum of skinfolds (1.01, 95\% CI 1.00-1.02, per mm), a history of GDM $(2.74,95 \%$ CI 1.66-4.50) and macrosomia (1.97, 95\% CI 1.03-3.98) [32•]. This led us to wonder whether women diagnosed early had likely mild hyperglycaemia before conception and therefore could be considered to have prevalent GDM, while women developing GDM de novo after this time had incident GDM. Further work is urgently needed into the genotype, phenotype and natural history of what seem to be two types of GDM.

\section{The Effect of Lifestyle Intervention on GDM Risk, Potential Limitations of Lifestyle Intervention and Neonatal Outcomes}

In the main lifestyle trial, the combined HE\&PA group had significantly lower gestational weight gain $(-2.02,95 \% \mathrm{CI}-$ 3.58 to $-0.46 \mathrm{~kg}$ ) compared with UC, which did not affect fasting glucose, HOMA-IR or birth outcomes [19••]. Gestational weight gain was not significantly different to UC in groups with HE or PA alone [19••]. The lifestyle intervention had significant effects on nutrition and PA in the participants. Greater moderate to vigorous physical activity (MVPA) was found in the PA group $(0.36,95 \%$ CI 0.13 to $0.60 \mathrm{METh} / \mathrm{wk}$ ) at 24-28 weeks of pregnancy and in HE\&PA sedentary behaviour was decreased at 24 to $28(-3.0,95 \% \mathrm{CI}$ -4.47 to $-0.45 \mathrm{METh} / \mathrm{wk})$ and 35 to 37 weeks $(-2.98,95 \%$ $\mathrm{CI}-5.29$ to $-0.67 \mathrm{METh} / \mathrm{wk}$ ) compared with UC. Nutrition improved in $\mathrm{HE}$ with a reduced intake of sugared drinks, carbohydrates and portion size and higher vegetable intake. Reduced portion size and higher vegetable intake were found in the combined HE\&PA group in comparison with UC [19••].

In a post hoc analysis, even a gestational weight gain limitation of $2.6 \mathrm{~kg}$ at $24-28$ and $4.3 \mathrm{~kg}$ at $35-37$ weeks of gestation (i.e. $6.7 \pm 5.9$ vs. $4.1 \pm 5.0$ and $11.3 \pm 6.7$ vs. $7.0 \pm$ $6.0 \mathrm{~kg}, p<0.05$ both) had no impact on metabolic outcomes but reduced the frequency of large for gestational age babies $(25 \%$ vs $16 \% p<0.05)$. [33•]. Increased fasting glucose was found in the HE group, and because less carbohydrate intake was reported in this group, higher lipolysis was speculated as a potential explanation for increased fasting glucose. Increased free fatty acid levels were also reported in the cord blood from the offspring of the HE group $(0.34 \pm 0.22$ vs. $0.29 \pm$ $0.16 \mathrm{mmol} / \mathrm{L} p=0.01)[34 \bullet \bullet]$. No maternal or fetal differences were found between the PA vs. no PA intervention groups $[34 \bullet \bullet]$.

In the combined HE\&PA group, an analysis of the effects of lifestyle intervention on offspring outcomes found lower adiposity (sum of skinfolds, fat mass and percentage and cord blood leptin levels) compared with UC [35••]. Lower cord blood leptin levels were also reported in female offspring in the PA group. A mediation analysis showed that the decrease in sedentary time in HE\&PA and PA groups mediated the intervention effects on neonatal adiposity. Interestingly, the reduction in GWG did not mediate these effects.

\section{Psychological Aspects}

In a further analysis, mediators of behaviour change were analysed and an increased task self-efficacy (defined as one's belief in one's ability to succeed in specific situations or accomplish a task) was identified to be the main element to improve health behaviour [36] The improvements in this factor had mediatory effects on physical activity and decreased intake of sugared drinks and portion size.

In a study testing the association of mental health status and MVPA or sedentary behaviour measured with accelerometers, $27.1 \%$ of the pregnant women had depressed mood with a WHO-5 well-being index score $<50$ before 20 weeks of gestation [37•]. Pregnancy-related worries and lower perceived social support were significantly higher in these women. Women with good mental health status at baseline spent 
significantly more time in MVPA compared with women with depressed mood (24 min vs. $13 \mathrm{~min} /$ day), whereas pregnancyrelated worries did not affect MVPA time. In an adjusted linear regression model, women with good mental health at baseline spent $85 \%$ more time doing MVPA compared with those with depressed mood $(p<0.05)$ [37•]. MVPA of at least $30 \mathrm{~min}$ was achieved by only $29.6 \%$ of women.

Before 20 weeks of gestation, several maternal characteristics were found to be associated with low mental health status [38]. European ethnicity, social support as well as selfefficacy were protective factors, whereas shift work, lack of sleep and pregnancy-related worries increased risk for low well-being in early pregnancy [38].

\section{Vitamin D and GDM Risk}

In the DALI Vitamin D trial, 25-hydroxyvitamin D baseline levels exceeding $50 \mathrm{nmol} / 1$ were reported across all study centres [25••], which was an unexpected finding as previous studies had suggested that a majority of obese pregnant women would have low vitamin D levels [39, 40]. Supplementation of 25-hydroxyvitamin D resulted in greater sufficiency in the vitamin D arm, with significant differences in levels compared with placebo at 24-28 (97 vs. 74\%) and 35-37 (98 vs. 78\%, $p<0.001$ both) weeks gestation. Interestingly, lower fasting glucose was found in the vitamin D group compared with placebo $(-0.14,95 \% \mathrm{CI}-0.28-0.00 \mathrm{mmol} / \mathrm{l})$ at $35-$ 37 weeks gestation; however, this difference was thought to be too small and too late to be clinically significant. No other metabolic or perinatal effects were found.

\section{Cost-effectiveness}

DALI evaluated cost-effectiveness of lifestyle intervention compared with UC in pregnancies at high risk for GDM and found that the HE\&PA intervention was the preferred prevention strategy for limitation of gestational weight gain at 3537 weeks, and was cost-effective for quality-adjusted life years (QALYs) after delivery [41•], with an average saving of €91,254 per QALY gained. No cost-effectiveness was found for any of the lifestyle interventions for fasting glucose or HOMA-IR at 35-37 weeks.

\section{Discussion}

The DALI lifestyle trial was one of 4 large RCTs to test whether GDM could be prevented by lifestyle interventions [17, 18, $20,24]$. The DALI vitamin D trial was one of only several trials to try to prevent GDM using vitamin D [25••]. The epidemiological, behavioural and pathophysiological evidence that the DALI studies have generated have added significantly to our understanding of GDM.

DALI demonstrated that in a multicentre, multicultural setting, the implementation of behavioural lifestyle intervention is challenging but possible. Coaches with good training can achieve beginning professional level quickly and are able to deliver motivational interviewing-based lifestyle intervention in a high quality with high fidelity. Nonetheless, there is a need for more information about the implementation of such strategies in diabetes prevention studies and the success of such interventions using coaches with limited experience of motivational interviewing.

\section{Women with Prevalent GDM}

A key strength of DALI is that it used uniform IADPSG/ WHO criteria in all centres across Europe from early pregnancy to 35-37 weeks. The trials were conducted having excluded those women with early (prevalent) GDM (i.e. a diagnostic OGTT on screening for the study). Subsequent to the commencement of the studies, the IADPSG determined that it was unclear which criteria should be used early in pregnancy. It may be that diagnostic thresholds should be higher (DALI excluded some women without GDM but at the higher end of the 'normal' glycaemic spectrum). DALI therefore was potentially limited by excluding women who might be considered not to have GDM once criteria at this early stage are developed. If so, such women would be at the top end of the glucose range, and may have benefited from lifestyle change. Using these IADPSG criteria, DALI was able to demonstrate a high prevalence of GDM in women with BMI above $29 \mathrm{~kg} / \mathrm{m}^{2}$ and high variation of GDM across Europe. Risk factors identified at several time points in pregnancy were mostly nonmodifiable. The studies have also shown the different proportions of women with prevalent GDM and the additional 40$60 \%$ developing GDM after excluding those with early GDM. Women with early/prevalent GDM clearly feature characteristics of the metabolic syndrome compared with women with GDM in later pregnancy. These data highlight the need for uniform diagnostic criteria and, if early treatment is shown to be beneficial, systematic screening starting in early pregnancy in high-risk groups [1•]. Nonetheless, the IADPSG/ WHO criteria were not validated for use in early pregnancy before 20 weeks of gestation and studies are ongoing to do so $[42,43]$. Results from the treatment of booking gestational diabetes mellitus (TOBOGM) pilot study indicate that the decision over which criteria to use may be complex, with early treatment resulting in less large for gestational age babies, but perhaps more babies requiring neonatal intensive care unit admission [44••]. 


\section{Comparison with Other Lifestyle Intervention Trials}

The DALI lifestyle intervention studies demonstrated that early behavioural intervention approaches were successful in reducing maternal weight gain significantly compared with prior intervention studies but did not have effects on glucose metabolism. Several large scale antenatal lifestyle intervention trials have been conducted aiming to prevent gestational diabetes mellitus (GDM) yielding conflicting results $[45 \bullet \bullet, 46 \bullet \bullet, 47 \bullet \cdot$ ]. Such trials have included high-risk populations including overweight or obese women or women with a history of GDM. The LIMIT and UPBEAT trials were the largest lifestyle intervention trials and included overweight or obese mothers [45••, 46••]. They used a combination of nutritional and physical activity interventions but were not able to reduce the risk of GDM development compared with the control group with standard antenatal care. Noticeable was the relatively low and non-significant difference in gestational weight gain between intervention and control group in LIMIT $(0 \mathrm{~kg})$ and UPBEAT $(-0.5 \mathrm{~kg})$, which might have not been enough to overcome insulin resistance in the intervention groups $[48,49]$. RADIEL was designed slightly differently with regard to the study population (overweight, many with a history of GDM) but also used healthy eating and physical activity intervention methods and reported a significant GDM reduction of $39 \%$ in the intervention group compared with the control group after correction for baseline parameters $[47 \bullet \bullet]$.

According to a Cochrane review, nutritional and exercise approaches have the potential to prevent GDM (RR 0.85, 95\% CI 0.71 to 1.01); however, a huge heterogeneity between studies, improper definition of interventions and description of behavioural change dynamics were reported, and there was no significant effect on GDM development [50]. An older systematic review of 29 lifestyle RCTs found a GDM risk reduction of $18 \%$ (RR $0.8295 \%$ CI $0.70-0.95$ ) with nutritional and exercise interventions overall. This reduction was primarily based on a significant risk reduction of GDM in studies with lifestyle interventions beginning before 15 weeks gestation (RR $0.78,95 \%$ CI 0.64 to 0.96 ), whereas no significant GDM risk reduction was seen thereafter (RR $0.97,95 \%$ CI 0.82 to 1.13 ) [51]. The DALI (vitamin D and lifestyle intervention in the prevention of GDM) trial was the first multicentre, randomised controlled study across 9 countries in Europe [17, 19••, 25••].

\section{Factors Affecting Lifestyle Intervention}

Egan and Simmons [8] discussed several aspects which might be relevant in the explanation of differences in outcomes of lifestyle trials. The heterogeneity of GDM itself with a variety of insulin secretory or insulin resistance or even mixed defects might be a cause of these differences, which needs further investigation [52]. Lifestyle changes are difficult to achieve and especially with increasing gestational week maintaining lifestyle goals might be hard. In a recent US study, below $10 \%$ of pregnant women managed to achieve the recommended PA of 5 days per week in the third trimester [53]. Weight gain is a relevant factor in GDM development. However, contrary to outside pregnancy, unlimited weight loss is not an option in pregnancy as this is related to fetal undernutrition and the potential programming of cardiometabolic disease and development of those in later adult life. Further research is needed in this field.

Psychosocial aspects are relevant factors in pregnancy and may have effects on lifestyle and thus lifestyle intervention. In general, a greater need for physical activity intervention is necessary in obese women with depression. Obesity, a depressed mood and an unhealthy lifestyle are potential additional risk factors for maternal and fetal pregnancy complications $[37 \cdot, 54]$. However, it is unclear if and to what extent lifestyle intervention can sufficiently improve MVPA in a group of depressed women as low mental health status may impede these ambitions. Thus, further research is needed to

Table 2 Summarized important lessons learned from the DALI trials

- High prevalence of GDM in early and throughout pregnancy in obese pregnant women using IADPSG/WHO 2013 criteria.

- GDM before 20 weeks of gestation was associated with features of the metabolic syndrome and insulin resistance compared with women with normal glucose tolerance.

- Risk factors for GDM vary between trimesters and were mostly of non-modifiable nature.

- Lifestyle intervention starting after the first trimester did not improve glucose levels or insulin resistance although significantly less weight gain was seen in the combined healthy eating and physical activity (HE\&PA) lifestyle intervention.

- Healthy behaviour increased in the lifestyle intervention groups.

- Mental well-being in pregnant women was associated with increased physical activity.

- Gestational weight gain reduction was not associated with improved glycaemic control but was associated with lower rates of babies born large for gestational age.

- The combined intervention of healthy eating and physical activity (HE\&PA) reduced fetal adiposity. This was mediated by a reduction in sedentary behaviour.

- Other mediating factors for improved maternal health behaviour were identified.

- The healthy eating intervention was associated with increased maternal and fetal free fatty acid levels possibly due to increased lipolysis and increased maternal fasting glucose.

- Cost-effectiveness was shown for HE\&PA - the intervention was cost-effective for quality-adjusted life years after delivery.

- Vitamin D supplementation had low, clinically insignificant effects on glucose levels and no effect on birth outcomes. 
investigate the effects of lifestyle intervention with regard to mental health status.

Taken together, DALI found that a lifestyle intervention was not able to prevent GDM from the second trimester but was cost-effective, limited gestational weight gain and improved pregnancy outcomes. A summary of important lessons learned from DALI is shown in Table 2. Several other interesting and useful findings will help to improve future intervention trials and the health outcome of mothers and their offspring.

Compliance with Ethical Standards The trial was registered under the number ISRCTN7059583, was performed according to the declaration of Helsinki and all local ethics committees approved the study.

Conflict of Interest Jürgen Harreiter, Gernot Desoye, Mireille van Poppel, Alexandra Kautzky-Willer, Fidelma Dunne, Rosa Corcoy, Roland Devlieger and David Simmons declare that they have no conflict of interest.

Human and Animal Rights and Informed Consent The studies included humans all of whom gave informed consent.

\section{Appendix The DALI core investigators group}

G. Desoye ${ }^{1}$, D. Simmons ${ }^{2}$, R. Corcoy ${ }^{3,4}$, J.M. Adelantado ${ }^{3}$, R. Devlieger $^{5}$, P. Damm ${ }^{6}$, E.R. Mathiesen ${ }^{6}$, D.M. Jensen ${ }^{7}$, L.L. Andersen $^{7}$, A. Lapolla ${ }^{8}$, MG. Dalfrà ${ }^{8}$, A Bertolotto ${ }^{9}$, E. Wender-Ozegowska $^{10}$, A. Zawiejska ${ }^{10}$, D J. Hill ${ }^{11}$, M.N.M. ${\text { van } \text { Poppel }^{12} \text {, F.J. Snoek }}^{13}$, A. Kautzky-Willer ${ }^{14}$, FP. Dunne ${ }^{15}$

Gernot Desoye, PhD ${ }^{1}$ Department of Obstetrics and Gynaecology, Medizinische Universitaet Graz, Graz, Austria. E: gernot.desoye@medunigraz.at

David Simmons, MD (Cantab) ${ }^{2}$ Institute of Metabolic Science, Addenbrookes Hospital, Cambridge, England and Macarthur Clinical School, Western Sydney University, Sydney, Australia. E: Da.simmons@westernsydney.edu.au

Rosa Corcoy, PhD ${ }^{3}$ Institut de Recerca de l'Hospital de la Santa Creu i Sant Pau, Barcelona, Spain; Departament de Medicina, Universitat Autònoma de Barcelona, Bellaterra, Spain.

${ }^{4}$ CIBER Bioengineering, Biomaterials and Nanotechnology, Instituto de Salud Carlos III, Zaragoza, Spain E: RCorcoy@santpau.cat

Juan M Adelantado, $\mathrm{PhD}^{3}$ Institut de Recerca de l'Hospital de la Santa Creu i Sant Pau, Barcelona, Spain. E: JAdelantado@santpau.cat
Roland Devlieger, PhD ${ }^{5} \mathrm{KU}$ Leuven Department of Development and Regeneration: Pregnancy, Fetus and Neonate. Gynaecology and Obstetrics, University Hospitals Leuven, Belgium. E: roland.devlieger@uzleuven.be

Peter Damm, DMsc. ${ }^{6}$ Center for Pregnant Women with Diabetes, Departments of Endocrinology and Obstetrics, Rigshospitalet, Institute of Clinical Medicine, Faculty of Health and Medical Sciences, University of Copenhagen, Copenhagen, Denmark. E: pdamm@dadlnet.dk

Elizabeth Reinhardt Mathiesen, DMsc. ${ }^{6}$ Center for Pregnant Women with Diabetes, Departments of Endocrinology and Obstetrics, Rigshospitalet, Institute of Clinical Medicine, Faculty of Health and Medical Sciences, University of Copenhagen, Copenhagen, Denmark. E: elisabeth.reinhardt.mathiesen@regionh.dk

Dorte Moeller Jensen, $\mathrm{PhD}{ }^{7}$ Department of Endocrinology and Department of Gynaecology and Obstetrics, Odense University Hospital, Department of Clinical Research, Faculty of Health Science, University of Southern Denmark, Odense, Denmark. E: Dorte.Moeller.Jensen@rsyd.dk

Lise Lotte T. Anderson, MD. ${ }^{7}$ Department of Endocrinology and Department of Gynaecology and Obstetrics, Odense University Hospital, Department of Clinical Research, Faculty of Health Science, University of Southern Denmark, Odense, Denmark. E: lise.lotte.andersen@rsyd.dk

Annunziata Lapolla, MD ${ }^{8}$ Universita Degli Studi di Padova, Padua, Italy. E: annunziata.lapolla@unipd.it

Maria G Dalfrà, MD ${ }^{8}$ Universita Degli Studi di Padova, Padua, Italy. E: u053734@sanita.padova.it

Alessandra Bertolotto, PhD. ${ }^{9}$ Azienda OspedalieroUniversitaria Pisana, Pisa, Italy. E: alessandrabertolotto1959 @yahoo.it

Ewa Wender-Ozegowska, PhD. ${ }^{10}$ Division of Reproduction, Poznan University of Medical Sciences, Poznan, Poland. E: ewaoz@post.pl

Agnieszka Zawiejska, PhD ${ }^{10}$ Division of Reproduction, Poznan University of Medical Sciences, Poznan, Poland E: agazaw@post.home.pl

David J.Hill, DPhil. ${ }^{11}$ Recherche en Santé Lawson SA, St. Gallen, Switzerland. E: david.hill@lawsonresearch.com

Mireille N.M. van Poppel, PhD. ${ }^{12}$ Department of Public and Occupational Health, EMGO ${ }^{+}$-Institute for Health and Care Research, VU University Medical Centre, Amsterdam, 
the Netherlands and Institute of Sport Science, University of Graz, Graz, Austria. E: mireille.van-poppel@uni-graz.at

Frank J Snoek, PhD ${ }^{13}$ Department of Medical Psychology, EMGO+-Institute for Health and Care Research, VU University Medical Centre and Medical Psychology AMC, Amsterdam, the Netherlands. E: fj.snoek@vumc.nl

Alexandra Kautzky-Willer, MD ${ }^{14}$ Gender Medicine Unit, Division of Endocrinology and Metabolism, Department of Medicine III, Medical University of Vienna, Vienna, Austria. E: alexandra.kautzky-willer@meduniwien.ac.at

Fidelma Dunne, $\mathrm{PhD}{ }^{15}$ National University of Ireland, Galway, Ireland. E: fidelma.dunne@nuigalway.ie

\section{References}

Papers of particular interest, published recently, have been highlighted as:

- Of importance

•. Of major importance

1. Buckley BS, Harreiter J, Damm P, Corcoy R, Chico A, Simmons D, et al. Gestational diabetes mellitus in Europe: prevalence, current screening practice and barriers to screening. A review. Diabet Med. 2012;29(7):844-54. Review showing inconsistencies regarding GDM diagnostic criteria as well as other aspects hampering GDM screening and treatment across Europe.

2. Simmons D. Diabetes and obesity in pregnancy. Best Pract Res Clin Obstet Gynaecol. 2011;25(1):25-36.

3. Ferrara A. Increasing prevalence of gestational diabetes mellitus: a public health perspective. Diabetes Care. 2007;30(Suppl 2):S1416.

4. Lavery JA, Friedman AM, Keyes KM, Wright JD, Ananth CV. Gestational diabetes in the United States: temporal changes in prevalence rates between 1979 and 2010. BJOG. 2017;124(5):804-13.

5. Bellamy L, Casas JP, Hingorani AD, Williams D. Type 2 diabetes mellitus after gestational diabetes: a systematic review and metaanalysis. Lancet. 2009;373(9677):1773-9. Systematic review describing the high risk of T2DM in women with a history of GDM.

6. Harreiter J, Dovjak G, Kautzky-Willer A. Gestational diabetes mellitus and cardiovascular risk after pregnancy. Women's Health (Lond Engl). 2014;10(1):91-108.

7. Retnakaran R, Shah BR. Role of type 2 diabetes in determining retinal, renal, and cardiovascular outcomes in women with previous gestational diabetes mellitus. Diabetes Care. 2017;40(1):101-8.

8. Egan AM, Simmons D. Lessons learned from lifestyle prevention trials in gestational diabetes mellitus. Diabet Med. 2019;36(2):14250 .

9.• Aroda VR, Christophi CA, Edelstein SL, Zhang P, Herman WH, Barrett-Connor E, et al. The effect of lifestyle intervention and metformin on preventing or delaying diabetes among women with and without gestational diabetes: the diabetes prevention program outcomes study 10-year follow-up. J Clin Endocrinol Metab. 2015;100(4):1646-53. Follow-up subanalysis of the DPP focussing on women with a history of GDM and describing the effects of lifestyle intervention, metformin versus placebo on diabetes risk after 10 years.

10. Alouki K, Delisle H, Bermudez-Tamayo C, Johri M. Lifestyle interventions to prevent type 2 diabetes: a systematic review of economic evaluation studies. J Diabetes Res. 2016;2016:2159890.

11. Li R, Zhang P, Barker LE, Chowdhury FM, Zhang X. Costeffectiveness of interventions to prevent and control diabetes mellitus: a systematic review. Diabetes Care. 2010;33(8):1872-94.

12. Ekmekcioglu C, Haluza D, Kundi M. 25-Hydroxyvitamin D status and risk for colorectal cancer and Type 2 diabetes mellitus: a systematic review and meta-analysis of epidemiological studies. Int $\mathbf{J}$ Environ Res Public Health. 2017;14(2):127. https://doi.org/10. 3390/ijerph14020127.

13. Rafiq S, Jeppesen PB. Is Hypovitaminosis D Related to incidence of Type 2 diabetes and high fasting glucose level in healthy subjects: a systematic review and meta-analysis of observational studies. Nutrients. 2018;10(1):59. https://doi.org/10.3390/nu10010059.

14. Lee CJ, Iyer G, Liu Y, Kalyani RR, Bamba N, Ligon CB, et al. The effect of vitamin D supplementation on glucose metabolism in type 2 diabetes mellitus: a systematic review and meta-analysis of intervention studies. J Diabetes Complicat. 2017;31(7):1115-26.

15. Pittas AG, Dawson-Hughes B, Sheehan P, Ware JH, Knowler WC, Aroda VR, et al. Vitamin D supplementation and prevention of type 2 diabetes. N Engl J Med. 2019;381(6):520-30.

16. Zhang Y, Gong Y, Xue H, Xiong J, Cheng G. Vitamin D and gestational diabetes mellitus: a systematic review based on data free of Hawthorne effect. BJOG. 2018;125(7):784-93.

17. Jelsma JG, van Poppel MN, Galjaard S, Desoye G, Corcoy R, Devlieger R, et al. DALI: vitamin D and lifestyle intervention for gestational diabetes mellitus (GDM) prevention: an European multicentre, randomised trial - study protocol. BMC Pregnancy Childbirth. 2013;13:142. https://doi.org/10.1186/1471-2393-13-142.

18. Vellinga A, Zawiejska A, Harreiter J, Buckley B, Di Cianni G, Lapolla A, et al. Associations of body mass index (maternal BMI) and gestational diabetes mellitus with neonatal and maternal pregnancy outcomes in a multicentre European database (diabetes and pregnancy vitamin $\mathrm{D}$ and lifestyle intervention for gestational diabetes mellitus prevention). ISRN Obes. 2012;2012:424010.

19.• Simmons D, Devlieger R, van Assche A, Jans G, Galjaard S, Corcoy R, et al. Effect of physical activity and/or healthy eating on GDM risk: the DALI lifestyle study. J Clin Endocrinol Metab. 2017;102(3):903-13. DALI main lifestyle intervention study describing effects of healthy eating, physical activity or a combined group versus usual care on gluco-metabolic outcomes in pregnancy.

20. Jelsma JGM, Simmons D, Gobat N, Rollnick S, Blumska K, Jans $\mathrm{G}$, et al. Is a motivational interviewing based lifestyle intervention for obese pregnant women across Europe implemented as planned? Process evaluation of the DALI study. BMC Pregnancy Childbirth. 2017;17(1):293

21. Simmons D, Jelsma JG, Galjaard S, Devlieger R, van Assche A, Jans $G$, et al. Results from a European multicenter randomized trial of physical activity and/or healthy eating to reduce the risk of gestational diabetes mellitus: the DALI lifestyle pilot. Diabetes Care. 2015;38(9):1650-6. Pilot study of the DALI trial testing the lifestyle interventions and study procedures.

22. IOM (Institute of Medicine) and NRC (National Research Council). Weight gain during pregnancy: reexamining the guidelines. Washington, DC: The National Academies Press. 2009. Criteria for recommended weight gain in pregnancy according to prepregnancy BMI.

23. Miller WR, Rollnick S. Motivational interviewing, preparing people to change addictive behavior. New York: The Guildford Press; 1991. 
24. Simmons D, Daniels T, Simmons DJ, van Poppel M, Harreiter J. A Mobile electronic record for lifestyle coaches in gestational diabetes prevention. JMTM. 2019;8(1):37-49.

25.• Corcoy R, Mendoza LC, Simmons D, Desoye G, Adelantado JM, Chico A, et al. The DALI vitamin D randomized controlled trial for gestational diabetes mellitus prevention: no major benefit shown besides vitamin D sufficiency. Clin Nutr. 2019; (in press). https:// doi.org/10.1016/j.clnu.2019.04.006. DALI vitamin D trial describing the effects of Vitamin D3 supplementation on gluco-metabolic outcomes in pregnancy.

26. Hollis BW, Johnson D, Hulsey TC, Ebeling M, Wagner CL. Vitamin D Supplementation during pregnancy: double-blind, randomized clinical trial of safety and effectiveness. J Bone Miner Res. 2011;26(10):2341-57. Vitamin D dosage and safety trial in pregnancy.

27. Ross AC, Manson JE, Abrams SA, Aloia JF, Brannon PM, Clinton SK, et al. The 2011 report on dietary reference intakes for calcium and vitamin D from the Institute of Medicine: what clinicians need to know. J Clin Endocrinol Metab. 2011;96(1):53-8.

28. Miller WR, Rollnick S. The effectiveness and ineffectiveness of complex behavioral interventions: impact of treatment fidelity. Contemp Clin Trials. 2014;37(2):234-41.

29. Jelsma JG, van Leeuwen KM, Oostdam N, Bunn C, Simmons D, Desoye G, et al. Beliefs, barriers, and preferences of European overweight women to adopt a healthier lifestyle in pregnancy to minimize risk of developing gestational diabetes mellitus: an explorative study. J Pregnancy. 2016;2016:3435791.

30.• Egan AM, Vellinga A, Harreiter J, Simmons D, Desoye G, Corcoy $\mathrm{R}$, et al. Epidemiology of gestational diabetes mellitus according to IADPSG/WHO 2013 criteria among obese pregnant women in Europe. Diabetologia. 2017;60(10):1913-21. DALI epidemiology paper describing GDM prevalence throughout Europe and risk factors.

31. Mendoza LC, Harreiter J, Simmons D, Desoye G, Adelantado JM, Juarez F, et al. Risk factors for hyperglycemia in pregnancy in the DALI study differ by period of pregnancy and OGTT time point. Eur J Endocrinol. 2018;179(1):39-49.

32. Harreiter J, Simmons D, Desoye G, Corcoy R, Adelantado JM, Devlieger R, et al. IADPSG and WHO 2013 gestational diabetes mellitus criteria identify obese women with marked insulin resistance in early pregnancy. Diabetes Care. 2016;39(7):e90-2. Features of the MetSy in obese pregnant women with GDM diagnosis before 20 weeks of gestation.

33. Simmons D, Devlieger R, van Assche A, Galjaard S, Corcoy R, Adelantado JM, et al. Association between gestational weight gain, gestational diabetes risk, and obstetric outcomes: a randomized controlled trial post hoc analysis. Nutrients. 2018;10(11):1568. Secondary analysis describing the effects of weight gain on gluco-metabolic as well as birth outcomes.

34.• Harreiter J, Simmons D, Desoye G, Corcoy R, Adelantado JM, Devlieger R, et al. Nutritional lifestyle intervention in obese pregnant women, including lower carbohydrate intake, is associated with increased maternal free fatty acids, 3-beta-hydroxybutyrate, and fasting glucose concentrations: a secondary factorial analysis of the European multicenter, randomized controlled DALI lifestyle intervention trial. Diabetes Care. 2019;42(8):1380-9. Secondary analysis describing the effects of lifestyle intervention on maternal and fetal lipid metabolism, increased lipolysis was observed, which was associated with increased fasting glucose.

35.• van Poppel MNM, Simmons D, Devlieger R, van Assche FA, Jans $\mathrm{G}$, Galjaard S, et al. A reduction in sedentary behaviour in obese women during pregnancy reduces neonatal adiposity: the DALI randomised controlled trial. Diabetologia. 2019;62(6):915-25. Beneficial effects of combined healthy eating and physical activity on neonatal adiposity intervention was observed.
36. van Poppel MN, Jelsma JGM, Simmons D, Devlieger R, Jans G, Galjaard S, et al. Mediators of lifestyle behaviour changes in obese pregnant women. Secondary analyses from the DALI lifestyle randomised controlled trial. Nutrients. 2019;11(2):311. https://doi. org/10.3390/nu11020311.

37. de Wit L, Jelsma JG, van Poppel MN, Bogaerts A, Simmons D, Desoye G, et al. Physical activity, depressed mood and pregnancy worries in European obese pregnant women: results from the DALI study. BMC Pregnancy Childbirth. 2015;15:158. Secondary analysis describing the associations of maternal well being and physical activity in pregnancy.

38. Sattler MC, Jelsma JGM, Bogaerts A, Simmons D, Desoye G, Corcoy R, et al. Correlates of poor mental health in early pregnancy in obese European women. BMC Pregnancy Childbirth. 2017;17(1):404.

39. Bodnar LM, Catov JM, Roberts JM, Simhan HN. Prepregnancy obesity predicts poor vitamin D status in mothers and their neonates. J Nutr. 2007;137(11):2437-42.

40. McAree T. Obesity and vitamin D deficiency - current concepts on their impact on pregnancy. Eur Endocrinol. 2013;9(2):125-7.

41. Broekhuizen K, Simmons D, Devlieger R, van Assche A, Jans G, Galjaard S, et al. Cost-effectiveness of healthy eating and/or physical activity promotion in pregnant women at increased risk of gestational diabetes mellitus: economic evaluation alongside the DALI study, a European multicenter randomized controlled trial. Int J Behav Nutr Phys Act. 2018;15(1):23. Cost-effectiveness analysis of the DALI interventions.

42. Simmons D, Hague WM, Teede HJ, Cheung NW, Hibbert EJ, Nolan CJ, et al. Hyperglycaemia in early pregnancy: the treatment of booking gestational diabetes mellitus (TOBOGM) study. A randomised controlled trial. Med J Aust. 2018;209(9):405-6.43.

43. McIntyre HD, sacks DA, Barbour LA, Feig DS, Catalano PM, Damm P, et al. Issues with the diagnosis and classification of hyperglycemia in early pregnancy. Diabetes Care. 2016;39(1):53-4.

44.• Simmons D, Nema J, Parton C, Vizza L, Robertson A, Rajagopal R, et al. The treatment of booking gestational diabetes mellitus (TOBOGM) pilot randomised controlled trial. BMC Pregnancy Childbirth. 2018;18(1):151. Pilot study of an ongoing trial testing effects of early GDM treatment versus late GDM treatment and the effects on pregnancy and birth outcomes.

45.• Poston L, Bell R, Croker H, Flynn AC, Godfrey KM, Goff L, et al. Effect of a behavioural intervention in obese pregnant women (the UPBEAT study): a multicentre, randomised controlled trial. Lancet Diabetes Endocrinol. 2015;3(10):767-77. Effects of lifestyle intervention in obese women on LGA infants and glucose metabolism in pregnancy.

46.• Dodd JM, Turnbull D, McPhee AJ, Deussen AR, Grivell RM, Yelland LN, et al. Antenatal lifestyle advice for women who are overweight or obese: LIMIT randomised trial. BMJ. 2014;348: g1285. Effects of lifestyle intervention in overweight/obese women on LGA infants and glucose metabolism in pregnancy.

47.• Koivusalo SB, Rono K, Klemetti MM, Roine RP, Lindstrom J, Erkkola M, et al. Gestational diabetes mellitus can be prevented by lifestyle intervention: the Finnish gestational diabetes prevention study (RADIEL): a randomized controlled trial. Diabetes Care. 2016;39(1):24-30. Effects of lifestyle intervention in overweight women or women with a history of GDM on glucose metabolism in pregnancy.

48. Harreiter J, Kautzky-Willer A. Sex and gender differences in prevention of Type 2 diabetes. Front Endocrinol. 2018;9:220. https:// doi.org/10.3389/fendo.2018.00220.

49. Simmons D, van Poppel MN. UPBEAT, RADIEL, and DALI: what's the difference? Lancet Diabetes Endocrinol. 2015;3(10): 761.

50. Shepherd E, Gomersall JC, Tieu J, Han S, Crowther CA, Middleton $\mathrm{P}$. Combined diet and exercise interventions for preventing 
gestational diabetes mellitus. Cochrane Database Syst Rev. 2017;11:CD010443.

51. Song C, Li J, Leng J, Ma RC, Yang X. Lifestyle intervention can reduce the risk of gestational diabetes: a meta-analysis of randomized controlled trials. Obes Rev. 2016;17(10):960-9.

52. Powe CE, Allard C, Battista MC, Doyon M, Bouchard L, Ecker JL, et al. Heterogeneous contribution of insulin sensitivity and secretion defects to gestational diabetes mellitus. Diabetes Care. 2016;39: $1052-5$.
53. Santo EC, Forbes PW, Oken E, Belfort MB. Determinants of physical activity frequency and provider advice during pregnancy. BMC Pregnancy Childbirth. 2017;17(1):286.

54. Barker ED, Kirkham N, Ng J, Jensen SK. Prenatal maternal depression symptoms and nutrition, and child cognitive function. $\mathrm{Br} \mathrm{J}$ Psychiatry. 2013;203(6):417-21.

Publisher's Note Springer Nature remains neutral with regard to jurisdictional claims in published maps and institutional affiliations.

\section{Affiliations}

\section{Jürgen Harreiter ${ }^{1} \cdot$ Gernot Desoye $^{2} \cdot$ Mireille N. M. van Poppel $^{3} \cdot$ Alexandra Kautzky-Willer $^{1} \cdot$ Fidelma Dunne $^{4}$. Rosa Corcoy ${ }^{5,6,7}$ • Roland Devlieger ${ }^{8}$. David Simmons ${ }^{9,10}$ • Juan M. Adelantado • Peter Damm • Elizabeth Reinhardt Mathiesen • Dorte Moeller Jensen - Lise Lotte T. Anderson • Annunziata Lapolla • Maria G. Dalfrà • Alessandra Bertolotto • Ewa Wender-Ozegowska • Agnieszka Zawiejska • David J. Hill • Frank J. Snoek • on behalf of the DALI Consortium}

Jürgen Harreiter

juergen.harreiter@meduniwien.ac.at

\section{Gernot Desoye}

gernot.desoye@medunigraz.at

Mireille N. M. van Poppel

mireille.van-poppel@uni-graz.at

Alexandra Kautzky-Willer

alexandra.kautzky-willer@meduniwien.ac.at

1 Gender Medicine Unit, Division of Endocrinology and Metabolism, Department of Medicine III, Medical University of Vienna, Vienna, Austria

2 Department of Obstetrics and Gynecology, Medical University of Graz, Graz, Austria

3 Institute of Sport Science, University of Graz, Graz, Austria

4 National University of Ireland, Galway, Ireland

5 Institut de Recerca de l'Hospital de la Santa Creu i Sant Pau, Barcelona, Spain

6 CIBER Bioengineering, Biomaterials and Nanotechnology, Instituto de Salud Carlos III, Madrid, Spain
Fidelma Dunne

fidelma.dunne@nuigalway.ie

Rosa Corcoy

rcorcoy@santpau.cat

Roland Devlieger

roland.devlieger@uz.kuleuven.ac.be

7

Departament de Medicina, Universitat Autònoma de Barcelona, Bellaterra, Spain

8 KU Leuven Department of Development and Regeneration: Pregnancy, Fetus and Neonate, Gynaecology and Obstetrics, University Hospitals Leuven, Leuven, Belgium

9 Institute of Metabolic Science, Addenbrookes Hospital, Cambridge, England

10 School of Medicine, Macarthur Clinical School, Western Sydney University, Locked Bag 1797, Campbelltown, NSW 2751, Australia 